\title{
Prenons-nous assez au sérieux le tsunami gris?
}

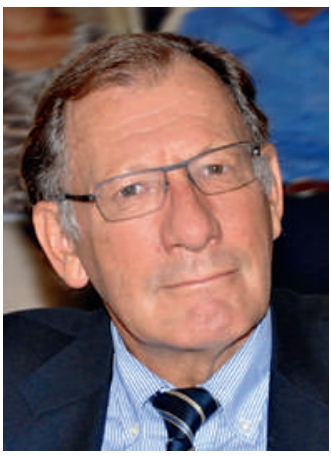

Jean Martin

1 Lüthi D. «Les personnes âgées sont particulièrement vulnérables». (entretien avec le Dr Stéfanie Monod). Bull Méd Suisses. 2014; 95(9):359-61.

2 La lettre de la Cohorte, $\mathrm{n}^{\circ} 10$, déc. 2013. www.lc65plus.ch

3 Fragnière JP. Une politique des âges et des générations. Sierre: Editions A la Carte; 2013.

4 Remarque finale: au vu des défis très pratiques que pose le vieillissement démographique, on reste songeur devant les espoirs suscités dans certains cercles par les discours faisant miroiter un allongement important de la vie humaine (pour bientôt?) voire l'immortalité (pour un peu plus tard). Vers une société de cauchemar où, si les gens ne meurent plus, de nouveaux arrivants (les enfants) ne seront pas du tout bienvenus. Brave new world...
On connaît les «Grey Panthers», mouvement lancé aux Etats-Unis au début des années 1970, seniors qui ont déployé une action politique en faveur des personnes âgées. De la même couleur (!), on parle maintenant du tsunami gris en référence aux défis que pose à toute la société (au système médico-social, à celui des retraites, etc.) le vieillissement démographique. Les pouvoirs publics ont à adapter leurs planifications en conséquence, mais les chiffres en cause reçoivent-ils l'attention voulue (je rappelle que, sous réserve de migrations fortes, les prévisions démographiques pour un pays donné sont solides, y compris à des années de distance)? Il apparait que le Gouvernement vaudois lui en tient compte, puisqu'il a nommé une médecin gériatre à la fonction lourde et exposée de cheffe du Service de la santé publique. Citation du Dr Stéfanie Monod: «Si notre système de santé ne se remet pas en question, nous ferons face à une catastrophe d'ici 20 à 30 ans. C'est certain. Une étude a même montré que le nombre de personnes de plus de 85 ans admises aux urgences du Centre Hospitalier Universitaire Vaudois à Lausanne (CHUV) a augmenté de près de 50 pour cent entre 2005 et 2010 [1]. Aujourd'hui, la plus grande partie de la médecine dite de famille est consacrée aux seniors; le modèle du médecin de premier recours qui s'occupait aussi bien des enfants que des grands-parents, qui faisait des accouchements et des actes chirurgicaux, a pratiquement disparu.

Pour étudier les bonnes mesures, il importe de disposer de données descriptives. A cet égard, des études de cohorte sont très utiles. On peut rappeler les précurseurs qu'ont été la Framingham Heart Study, lancée en 1948 près de Boston, la Whitehall Study suivant des fonctionnaires britanniques dès 1967, plusieurs cohortes de personnes touchées par le VIH, y compris en Suisse. S'agissant d'étude du vieillissement, la «Lausanne cohorte 65+» (Lc65+) a fêté récemment ses 10 ans [2]. C'est une plateforme de recherche qui a deux objectifs: mieux connaître les besoins pour adapter le système de santé, étudier le processus de fragilisation chez les personnes âgées. De son dernier bulletin, on tire que ses membres, qui avaient 65-70 ans en 2004, continuent pour les deux tiers d'entre eux à se considérer en bonne ou très bonne santé huit ans plus tard; au premier rang des problèmes viennent l'hypertension et l'arthrose; les facteurs qui leur donnent du souci sont d'abord la sécurité dans la rue et les ressources financières. Un peu plus d'un quart sont considérés comme pré-fragiles. Le "phénotype de fragilité» est nettement moins bon pour ceux en situation économique défavorable (confirmation bien sûr d'une constante de santé publique). S'agissant de l'éventualité d'entrer en EMS, leur premier souhait est de disposer d'une chambre et de sanitaires privatifs.

Ici, une «minute anthropologique»: on évoque souvent le respect, l'autorité, dont les anciens jouissent en milieu traditionnel, par exemple en Afrique - manifestation de ce qu'ils jouent encore un rôle d'importance dans la société. La diminution de cette «révérence» que l'on observe dans les sociétés industrialisées a plusieurs raisons. Celles et ceux qui atteignent un grand âge ne sont plus du tout exceptionnels (et on ne pense plus qu'ils y arrivent parce que «bénis des dieux»). Aussi, les anciens ne sont plus des références au plan des compétences techniques, dépassés qu'ils sont par les potentialités immenses ouvertes par l'électronique. Certains semblent prêts à mettre en cause le contrat intergénérationnel qui vaut depuis les années 1950. Chacun est sensibilisé aux menaces sur les régimes que l'Etat social et solidaire a pu mettre en place pour les seniors (AVS pour commencer, réseau de soins à domicile et EMS...) et à l'éventualité que les générations plus jeunes ne bénéficient pas de prestations au même niveau. Le risque de «fracture» est aggravé par la croissance des inégalités de ressources, absolues et/ ou relatives, qui se marquent dans le monde entier, entre les pays et au sein des pays.

Prenons-nous suffisamment au sérieux ces évolutions? Je ne parierais pas (y compris parce que personne n'aime les nouvelles gênantes). Certains s'y attachent [3]. S. Monod: «Il faut toujours garder en tête cette question: cette mesure et cette avancée technologique est-elle justifiée parce qu'elle améliore la survie et la qualité de vie des patients? La neurochirurgie robotique ou la protonthérapie ont leur place mais je pense en priorité aux centaines de personnes pour lesquelles nous n'avons pas de place ou de lit dans une institution adaptée.»

Je formule pour notre collègue gériatre qui devient patronne, gestionnaire, planificatrice, négociatrice/diplomate, acteur sociétal, des vœux chaleureux dans sa nouvelle activité. Bernanos: «On ne subit pas l'avenir, on le fait» [4].

Jean Martin, membre de la rédaction 